\title{
Leveraging Technology in Post-Disaster Settings: the Role of Digital Health/Telemental Health
}

\author{
Eugene F. Augusterfer ${ }^{1} \cdot$ Richard F. Mollica $^{2} \cdot$ James Lavelle $^{1}$
}

Published online: 28 August 2018

(C) Springer Science+Business Media, LLC, part of Springer Nature 2018

\begin{abstract}
The Purpose Review This paper will review the literature on global disasters and the mental health impact of disasters, and discuss the use of digital health/telemental health in providing care in post-disaster settings.

Recent Findings Global disasters, natural and manmade, are on the rise. As a consequence, there are increases in the health and mental health impact in the affected populations. We examine the literature on the health and mental health impact of disasters and the role of digital health/telemental health in response to meeting those needs. We examine the use of digital health/telemental health in two case examples, one of a natural disaster and one of a man-made disaster. Finally, we examine a blended telemental health model for collaboration between mental health and primary care providers in post-disaster settings.

Summary Digital health/telemental health is positioned on the cusp of the technology explosion, thus bringing much needed medical and mental health care to previously under-served populations.
\end{abstract}

Keywords Digital health $\cdot$ Telemental health $\cdot$ Telepsychiatry $\cdot$ Mental health $\cdot$ Disaster response $\cdot$ PTSD $\cdot$ Depression

\section{Introduction}

Advances in technology have allowed advanced specialized technology to be used in health care for diagnosis, treatment, and provider-to-provider consultation without regard for distance, thus bringing medical care to previously difficult-toreach populations and globally underserved populations. Digital health, telemedicine, telepsychiatry, and telemental health, hereinafter referred to as TMH, are a direct outgrowth of these advances in communications technology. TMH is an important component in meeting critical health and mental health needs of the global population, including those who have suffered from disasters, natural or man-made. The urgent

This article is part of the Topical Collection on Psychiatry in the Digital Age

Eugene F. Augusterfer efa@gmhnet.com

1 Harvard Program in Refugee Trauma, Harvard Global Mental Health Program, 22 Putnam Avenue, Cambridge, MA 02139, USA

2 Harvard Program in Refugee Trauma, Massachusetts General Hospital, Harvard Medical School, 22 Putnam Avenue, Cambridge, MA 02139, USA need for health care, including mental health care, to address the trauma caused by disasters is well documented. The United Nations reports that disasters, natural and man-made, have caused 68.5 million forcibly displaced persons, 40.3 million internally displaced persons, 25.4 million refugees, and 10 million stateless persons worldwide $[1 \bullet \bullet$. Thus, mass migration has contributed to geopolitical instability and rendered large numbers of displaced persons in need of medical and mental health care.

Natural disasters and armed conflict have marked human existence from time immemorial, but in recent times, the scale and scope of these events have increased markedly. Between 1994 and 2013, natural disasters have affected about 218 million people every year [2]. Mental health is an issue of global importance, with an estimated 300 million people worldwide suffering from depression, making it the leading cause of disability worldwide, and it is a major contributor to the overall the global disease burden $[3 \cdot \bullet]$. The rates of mental health illness in man-made and natural disasters are dramatically increased against an already existing baseline. The World Bank reports that in the presence of conflict situations, the psychiatric literature estimates an increase in disorder prevalence explained by high levels of stress that can prompt psychosocial or psychiatric disorders that were previously nonexistent or dormant $[4 \cdot \bullet]$. 
Since a disproportionate percentage of the impacted population are in low resource and difficult to reach settings, TMH can bring the promise of much needed specialty expertise to those in underserved and difficult to reach settings.

This article will discuss the impact of disasters, natural, man-made, and technological on the health and mental health of survivors and the role of TMH in meeting the ongoing needs of disaster survivors.

\section{Impact of Disasters}

Disasters, both natural and man-made, are on the rise worldwide, as documented by the Centre for Research on the Epidemiology of Disasters [5] (see Fig. 1).

As a consequence of the rise in disasters, humanitarian, health, and mental health needs in post-disaster settings are on the rise as well. As of June 2018, UNHCR reported 68.5 million forcibly displaced people worldwide, with $86 \%$ of the displaced people coming from low-and-middle income countries (LMICs) [6].

The need for health and mental health services to address the problems caused by disasters and displacement is well documented. Unipolar depression is number three on the World Health Organization Burden of Disease "Disability Adjusted Life Years (DALYs)" list of global disorders and is number one for women in the 15- to 44-year-age group worldwide. Further, based on current trends, unipolar depression is predicted to be the number one on the burden of disease worldwide by 2030 [7]. Disasters, natural or manmade, impact every person who is directly or indirectly involved, and many may need mental health support and/or treatment. Let us examine the most frequent mental healthrelated problems following a disaster. While both natural disasters and man-made disasters have impact on the health and mental health of the affected populations, there are differences in the scope and scale of natural disasters vs. manmade disasters.

\section{Mental Health Impact of Disasters}

As stated earlier, disasters, both natural and man-made, affect millions of people around the world every year. Mollica et al. report that mental health is becoming a central issue for public health complex emergencies [8•]. Although temporary

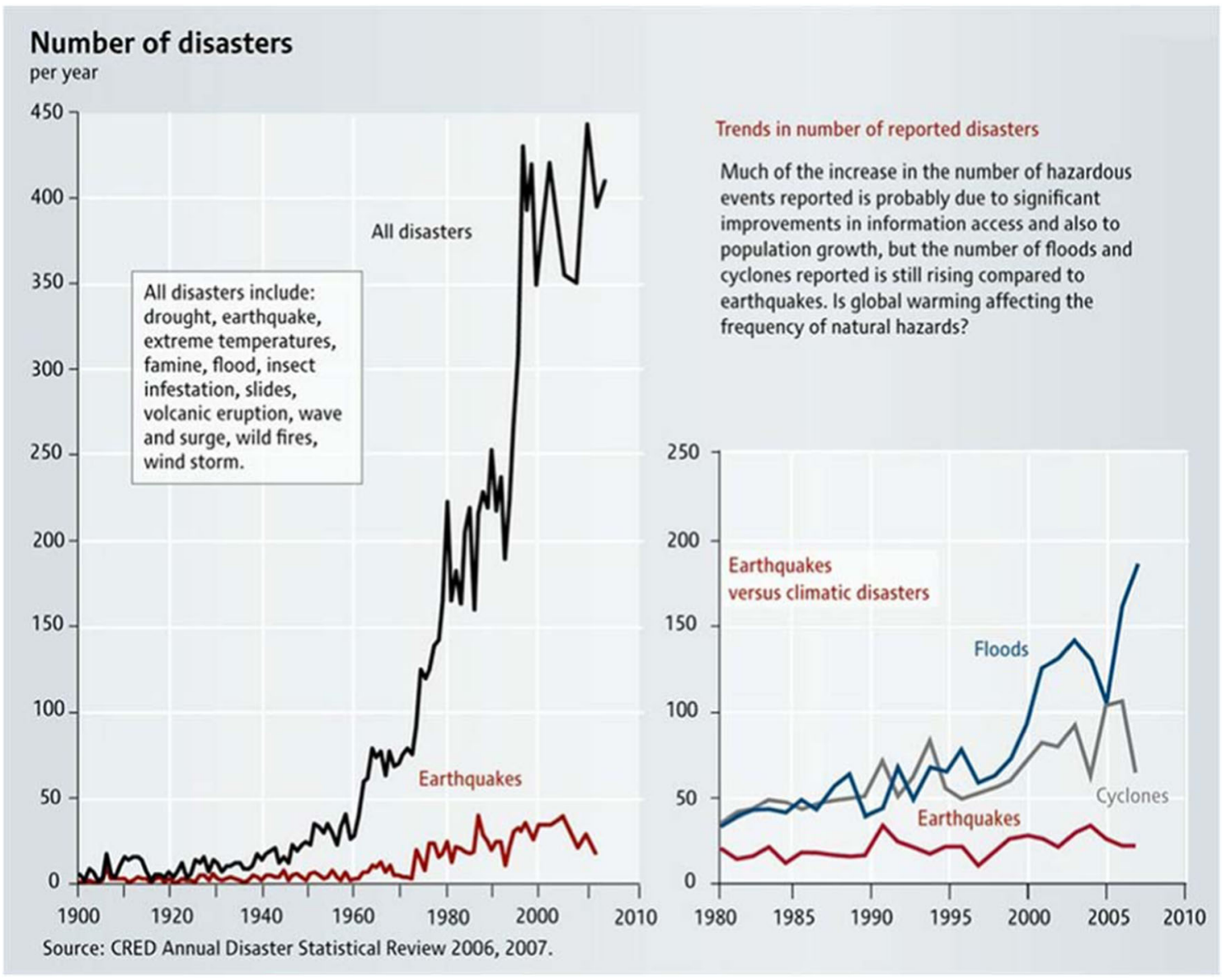

Fig. 1 Source: CRED Annual Disaster Statistical Review 2006, 2007 
symptoms tend to be common in the acute phase of disaster recovery, psychological sequelae can persist for up to 3 to 5 years after a natural disaster. In recent years, there have been a number of examples of large-scale disasters with multiple etiologies, both natural and man-made. A common thread in all of these disaster events is their significant impact on the health and mental health of survivors. As such, a percentage of the post-disaster population will develop mental health problems, such as post-traumatic stress disorder (PTSD), depression, anxiety, and other mental health conditions.

\section{Review of the Literature on Mental Health Impact of Disasters}

Neria and colleagues performed a systematic review of 284 peer-reviewed published studies of the mental health impact of natural disasters and concluded that "PTSD among persons exposed to disasters is substantial." And that the most consistently documented determinant of the risk of PTSD across the studies examined was the magnitude of exposure to the event [9]. Moscona et al. compared medical records at Tulane University Health Sciences Center from 2 years before Hurricane Katrina to 10 years after Katrina to determine health impact of Katrina. Comparing medical records pre-Katrina and post-Katrina, significant increases in various medical illnesses, including:

- coronary artery disease (pre $36.4 \%$ vs. post $47.9 \%$ )

- diabetes (pre $31.3 \%$ vs. post $39.9 \%$ )

- drug abuse (pre $10.2 \%$ vs. post $15.4 \%$ )

- psychiatric illness (pre 6.7\% vs. post $14.9 \%$ ) [10॰]

Dai et al. preformed a meta-analysis of 46 published articles which examined PTSD rates in 76,101 survivors of earthquakes who met the inclusion criteria. Using a random effects model, the combined incidence of PTSD after earthquakes was $23.66 \%$ [11].

The Great East Japan Earthquake of 2011, GEJE (9.1 magnitude), represents a combination of a natural disaster and a technological disaster. The undersea earthquake triggered a tsunami, which in turn caused a technological disaster (the meltdown of the nuclear reactors). Murakami et al. examined the records of 4311 residents in the tsunami zone (Miyagi Prefecture) 7 to 19 weeks following the disaster and found significant increases in blood pressure, high risk for cardiac event, and an increase in cases of sudden death [12]. In another study of the impact of the GEJE, Tsujiuchi et al. interviewed 2011 survivors of the GEJE (only 350 survivors met criteria for inclusion in the study) 1 year following the disaster and found that $59.4 \%$ met the criteria for probable PTSD using the IES-R (Japanese version) [13].

Another important area of concern following disasters is the risk of suicide. Alfanso studied suicide rates in Puerto
Rico before and after Hurricane Maria (2017) and reports a $32 \%$ increase in suicides (19 per month pre-Maria vs. 25 per month post-Maria) [14.•]. In another study, Kessler et al. examined PTSD, depression, suicidal ideation, and report that suicides increased from the original evaluation 8 months after Hurricane Katrina to the follow-up evaluation 1 year after the initial evaluation. Specifically, PTSD rates increased significantly from the initial evaluation to the follow-up evaluation, from 11.8 to $20.0 \%$. Additionally, suicidal ideation and suicide plans also increased significantly from the initial evaluation to the follow-up evaluation, suicidal ideation from 2.8 to $6.4 \%$, and suicide plans from 0.2 to $0.8 \%$ [15].

Man-made disasters, such as armed conflict, add another dimension that must considered, that is, an important factor for the survivors is knowing that the perpetrator intentionally inflicted the trauma. Survivors of these disasters have often endured unspeakable trauma to themselves and/or their loved ones. As such, the trauma tends to be more complex and thus the treatment response needs to address the complex nature of the trauma. In a study of Syrian refugees living in a UNHCR refugee camp in Turkey, Jefee-Bahloul et al. surveyed 354 refugees using the "HAD Stress" screening instrument and found that $41.8 \%$ of those surveyed scored consistent with PTSD [16•].

\section{The Role of Technology in Responding to Disasters}

As stated in the introduction, TMH through the use of digital technology bring the promise of specialty medical and mental health expertise to distant under-served populations and difficult to reach locations in the matter of seconds, thus providing much needed expertise in critical settings. As Frances Cairncross put it, we are now living in the time of "The Death of Distance" [17].

A disproportionately large percentage of global disasters occur in low-resource settings in the LMICs in the Developing World. As such, it is important to note that in the Developing World, specialty medical care is rare, especially mental health care. For example, following the 2010 earthquake in Haiti, 7.0 magnitude, which killed over 200,000 people and caused significant personal injuries and a significant displacement of the population, there were only 10 psychiatrists serving a population in excess of 10 million people [18].

As stated earlier, global disasters are on the rise, especially in low-resource settings, causing a significant increase in mental health-related problems, alongside a scarcity of mental health professionals. So what are the possible solutions? Digital Health and TMH bring the promise of mental health support in such settings.

While the peer-reviewed literature on the use of TMH in post-disaster settings is sparse, here are some pertinent articles. Augusterfer et al. reviewed the literature on the use of TMH in 
international and post-disaster settings and found that TMH can significantly enhance the delivery of mental health care in postdisaster settings [19]. Qadir et al. reported on the use of telepsychiatry in Pakistan following two natural disasters, floods in March 2015 followed by an earthquake, which measured 7.5 on the Richter scale, in October of 2015. Since these occurred in a remote area of Pakistan, mental health care was not readily available. Thus, a telepsychiatry network was established. The authors report that while concrete evidence regarding efficacy of telepsychiatry in this situation is still being assessed, it is definitely beneficial when in-person interaction between doctor and patient cannot occur [20]. For man-made disasters, such as the Syrian conflict, nearly half of the Syrian population has been displaced to refugee camps and other locations. These refugees who have been affected by conflict have heightened the risk of trauma-related disorders, such as PTSD, depression, anxiety disorders, and other reactions. Since mental health care is limited in refugee camps, Jefee-Bahloul et al. surveyed Syrian refugees in a refugee camp in Turkey and found that nearly half of those surveyed were interested in receiving mental health services through telepsychiatry [16•]. Yellowlees et al. found that telepsychiatry has the potential to improve access to psychiatric care in the event of a natural or man-made disaster [21].

\section{TMH Collaboration Model}

Given that mental health resources are often limited in postdisaster settings, a model that is particularly useful is a collaboration model of local (on-the-ground) primary care and distant mental health experts in a blended primary care - the TMH collaboration model. This model allows for the local primary care providers to collaborate with a distant mental health expert (psychiatrist or other mental health professional) regarding best practices in evidence-based mental health care for a patient in need. An important component of this model is that the local provider is part of the local culture; thus, he/she understands the language and importantly, the local expression of pain and suffering in the local culture. Also, most often, the local provider has an already established trusting relationship with the local population. These components cannot be stressed enough in terms of effective and appropriate delivery of care in post-disaster settings. Thus, the distant mental health expert is acting as a collaborative partner and advisor to the local primary care provider. This model has proven to be extremely successful and beneficial in lowresource settings, including post-disaster settings.

An example of this collaboration model is the model used by the Harvard Program in Refugee Trauma, HPRT, Global Mental Health Program (see Fig. 2). The HPRT model has been developed over $25+$ years of working in post-disaster settings. The model consists of a blended telemedicine program combining face-to-face collaboration between the mental health team and the on-site primary care team, followed by regularly scheduled telemedicine sessions (encrypted video-conferencing) that utilize the case study method. These sessions allow for the primary care providers to present difficult and/or challenging cases to the mental health expert team in a supportive peer-to-peer relationship of teaching/ supervision and mentoring model. This model is similar to the consultation-liaison model used in most academic teaching hospitals. HPRT has used this model successfully in over 85 countries over multiple years, thus building a strong global network.

Below is a pictorial example of the HPRT model. Of particular note is the arrow going both ways between primary care and mental health, representing the ongoing collaboration.

Before discussing some of the challenges in developing and implementing such model, let us review a couple of case examples.

\section{Case Example}

\section{Norcia Italy 6.5 Magnitude Earthquake}

On October 30, 2016, a 6.5 magnitude earthquake struck the town of Norcia in the central Apennine Mountains of Italy (see Fig. 3). The earthquake caused significant damage to property, but fortunately there were no deaths reported. The lack of deaths in Norcia was due, in large part, to the evacuation and displacement of a significant portion of the population months earlier due to strong tremors in Norcia following an earthquake in the neighboring town of Amitrice. The Amitrice earthquake (August 2016) caused 300 deaths, severe property damage, and displacement of the majority of survivors. Thus, when the earthquake hit Norcia, many residents had been relocated to safer areas. Nonetheless, the town center, which had been the hub of life in Norcia, was determined to be uninhabitable. As such, the sense of connectedness with friends and neighbors was severely impacted. As is noted in various studies, forced displacement of people and splitting of a community have significant impact as the "second wound" causing disruption of tightly knit communities often leading to additional trauma and instability of the impacted population, such as loss of permanent housing, families being separated, worries about livelihood or work, and loss of social ties. As an example, Munro et al. report a significant association between displacement after the 20132014 floods in the UK and the prevalence of symptoms of depression, anxiety, and post-traumatic stress disorder 1 year after the event [22•]. Following the emergency phase of the Norcia earthquake recovery, the HPRT team, which includes psychiatrists, psychologists, and clinical social workers, was invited to Norcia for face-to-face meetings with the local primary care medical leadership team to determine if a collaboration would be helpful. After several meetings, it was determined that 
Fig. 2 HPRT collaboration model (source: HPRT)

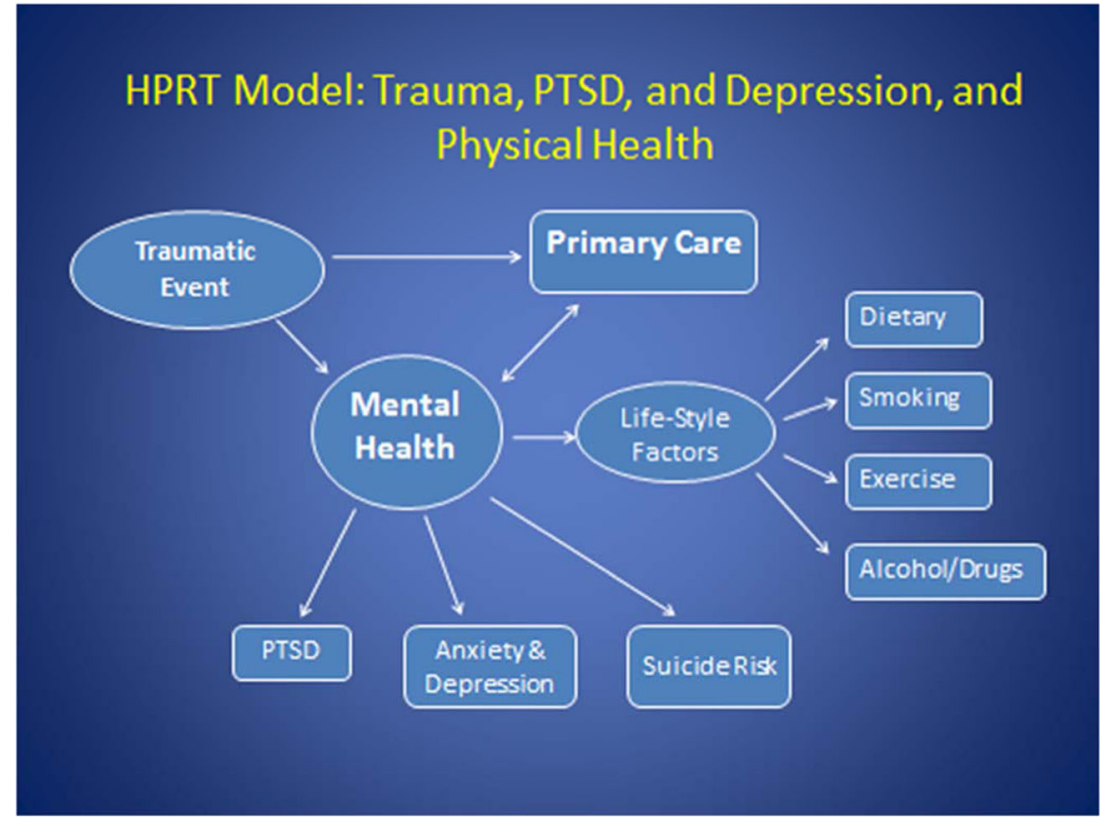

an ongoing collaboration would be initiated, which included a blended program of periodic face-to-face meetings with regularly scheduled distance telemedicine/video-conference meetings focused on case presentations of difficult and/or challenging cases to be presented by the primary care team for discussion and mentoring by the mental health team. It is very important to note that the mental health team is working with and through the primary care team as they are an integral part of a local community; thus, they have the cultural knowledge, language skills, and understanding of their patient population. Another important point regarding collaborating with a trusted local partner is that the local partner can negotiate with local officials regarding working in the local community and take the lead with securing local permission for any work that is planned and to be implemented. As an example, rebuilding a much needed local clinic and/or community center may need written permission, permits, and licenses, etc., from multiple layers of local officials.

The digital/TMH connection was through a vendor that supplies encrypted video-conferencing over Internet Protocol

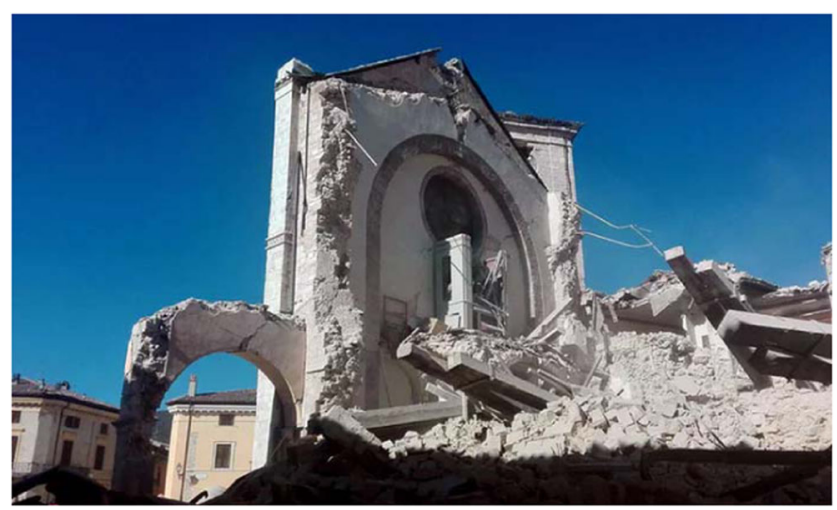

Fig. 3 Norcia, Italy, earthquake (source: HPRT)
(IP), allowing participants to call in from the location of their choice, including mobile cell phones and/or smartphones. This mental health-primary care collaboration model is highly recommended as it is a proven system that has worked very well in varied settings.

\section{Case Example}

\section{Man-Made Disaster (Syrian Conflict)}

The Syrian conflict is one example of a man-made disaster forced displacement of an estimated 5.6 million Syrians, an additional 6.6 million Syrians being internally displaced persons (IDPs), within Syria, and over 12 million people being left in need of medical and/or emotional care in Syria [23]. With no end to the conflict in sight, there is an urgent need for medical and mental health care for the survivors, IDPs, and those who have fled the country. As stated earlier, survivors have the additional complexity of knowing that the trauma they have suffered was inflicted willfully; thus, the trauma is often more complex and treatment must take this into consideration.

If communication systems have not been destroyed in combat, generally, they can be used to support digital health/TMH. However, in active combat settings, particular attention needs to be paid to addressing the security of the lines of communications, (highly encrypted signals) including digital health/ telemedicine, as non-secured communications can be intercepted by armed combatants and the information can be used to target hospitals, clinics, medical teams, and innocent survivors, as has been reported in the Syrian conflict. (armed conflict) which has caused more than 400,000 deaths, 
In conflict settings such as the Syrian crisis, Nassan et al. report that the provision of services with new technological resources such as telepsychiatry-defined as a "form of video conferencing that can provide psychiatric services to patients living in remote locations or otherwise underserved areas" - is being utilized and is of great value [24•]. In another study in the Syrian conflict zone, Jefee-Bahloul et al. report on the use of telepsychiatry in which the UK Internet-based program (The Syrian Telemental Health Network) provides training and clinical consultations in Syrian conflict settings. This "non-live" (i.e., asynchronous) platform allows referring providers (e.g., physicians, psychologists, nurses, non-specialized health care workers) to send data-safe clinical material about certain cases to specialists within the network to obtain specialized consultation and then supervision about their cases [25].

Also, unlike natural disasters, armed conflicts have a number of additional factors to be considered, such as the safety of the survivors and the medical and mental health teams.

\section{Recommendations}

There are a number of factors to consider in developing and implementing a digital health/TMH program in a post-disaster setting, to include:

- Having permission to work in the disaster area. Generally, in a foreign country, this would include an agreement with the Ministry of Health. Following the Haiti Earthquake of 2010, many well-meaning groups entered Haiti without specific permission and proceeded to violate local customs and, at times, local laws [26].

- Having a trusted local partner! This is important as many levels including understanding the language and culture of the impacted population, to include the language used to describe pain and suffering. Also, as stated above, a trusted local partner can negotiate with local officials for needed permissions, permits, licenses, etc.

- Having a clear agreement/protocol regarding scope and scale of the engagement, to include roles and responsibilities

- Based on in-depth collaboration with the local medical partner, having a clear understanding of the specific needs and the post-disaster phase of recovery. For example, in the emergency phase, Psychological First Aid is the generally accepted method of intervention, whereas mental health issues, such as depression, anxiety, PTSD, and other disorders, generally surface after the emergency phase, possibly 3 to 9 months after the disaster. Thus, ongoing collaboration with the local primary care providers by mental health experts is a valuable addition to the recovery plan in a postdisaster setting. Brandon et al. studied the efficacy of telehealth as a medium to train providers in distant locations and concluded that "telehealth represents a feasible avenue for training and supporting leaders of psychosocial interventions. In addition, telehealth is particularly well suited to the need for training providers in areas outside urban centers or academic communities" [27•]

- Having a clear understanding of the status of the local telecommunications system, that is, what works and what does not, including the possibility of telecom system being damaged in the disaster. Generally, local governments and businesses are eager to restore the telecommunications system for their use. As such, once the system is restored, telemedicine linkage is possible, including $m$ Health (cellphone and smartphone use). It is also extremely important to insure, as best possible, that the communications system that is used for the digital health/telemedicine network is secure and encrypted. Again, a trusted local partner should be able to provide reliable information regarding the status of the telecommunications system.

- An often overlooked factor is providing care for those on the front lines of disaster relief and recovery, including first responders. Medical and mental health teams are often pushed to their limits and are therefore subject to burnout and compassion fatigue. Mollica et al. report that all mental health providers should be provided a self-care program that includes identification of risk factors and opportunities for resiliency to prevent negative mental health outcomes [28•]. Garbern and colleagues performed a systematic review of 2849 abstracts and 66 full-length articles focused on outcomes among disaster and humanitarian responders. The majority of the research on health outcomes focused on mental health with a particular focus on post-traumatic stress disorder (PTSD) and depression. PTSD rates ranged from 0 to $34 \%$, and depression rates ranged from 21 to $53 \%$. They concluded that mental health disorders among responders are higher and vary more than previously thought [29•].

- An extremely important factor is to consider partnering with a well-established trusted organization. In doing so, the organization will provide needed structure and support. Most organizations welcome qualified volunteers who are able to help meet the needs of disaster survivors. Entering a post-disaster setting as a "lone wolf" is highly discouraged!

\section{Conclusion}

In summary, disasters, natural and man-made, are on the rise. As such, the need for medical and mental health care in postdisaster settings is substantial and escalating. Given the increase in global disasters, the need for qualified mental health specialists to assist in the care of survivors is great. Advances in digital technology, including the use of mobile phones [30•], allows for global real-time communications, which in 
turn allows for medical care to be rendered quickly without restrictions for location. Thus, TMH is an important component of post-disaster mental health care.

However, as stated above in recommendations, there are a number of factors to consider prior to providing medical care and services in a post-disaster setting. While all of the points in the "Recommendations" section are important, there are several crucially important points that need to be considered, including:

- working with a trusted local partner

- having a clear written agreement as to scope, scale, and responsibilities

- collaborating with a trusted established organization, not solo as a "lone wolf," which can be ineffective and possibly dangerous

Additionally, the authors make a strong plea for the publication of one's work related to the use of TMH in disaster settings as the published literature on the subject is still too sparse. To that point, Latifi and Tilley reviewed the literature on the use of telemedicine in post-disaster settings from January 1980 to September 2013, and reported that 17,565 disasters were recorded, including natural and man-made disasters, yet only 878 peer-reviewed articles and chapters on the use of telemedicine were published [31].

In conclusion, digital health and TMH are on the cusp of the technology explosion that is bringing new avenues to reach global populations in need of medical and mental health care regardless of location.

Acknowledgments The authors would like to acknowledge our gratitude to Barbara M. Peyser, PsyD, for her ongoing support, reading, and editing of this manuscript. We are truly grateful for the kind manner in which she read, edited, and re-read this paper.

\section{Compliance with Ethical Standards}

Conflict of Interest Eugene F. Augusterfer, Richard F. Mollica, and James Lavelle declare no conflict of interest.

Human and Animal Rights and Informed Consent This article does not contain any studies with human or animal subjects performed by any of the authors.

\section{References}

Papers of particular interest, published recently, have been highlighted as:

- Of importance

-• Of major importance

1.• United Nations High Commissioner on Refugees, Global trends in forced displacement, 19 June 2018. This report documents the current increase in the number of globally displaced persons.
2. Centre for Research on the Epidemiology of Disasters. The human cost of natural disasters, a global perspective. 2015.

3.• World Health Organization. Depression fact sheet, 22 March 2018. This report documents the increase in Depression world-wide.

4.• World Bank Report on Psychosocial Support in Fragile and Conflict-Affected Settings, May 2016 http://www.worldbank.org/ en/topic/fragilityconflictviolence/brief/psychosocial-support-infragile-and-conflict-affected-settings. This report documents the increase in psychiatric disorders in man-made disasters - conflict situations.

5. Centre for Research on the Epidemiology of Disasters. Annual Disaster Statistical Review 2016.

6. United Nations High Commissioner on Refugees. Global Trends in Forced Displacement, 19 June 2018.

7. World Health Organization. Depression fact sheet, 22 March 2018.

8. Mollica RF, Lopes Cardozo B, Osofsky HJ, Raphael B, Ager A, Salama P. Mental health in complex emergencies. Lancet. 2004;364(9450):2058-67. This study demonstrates the impact of public health complex emergencies on the mental health of survivors.

9. Neria Y, Nandi A, Galea S. Post-traumatic stress disorder following disasters: a systematic review. Psychol Med. Apr 2008;38(4):46780 .

10. Moscona JC, Peters MN, Maini R, Katigbak P, Deere B, Gonzales $\mathrm{H}$, et al. The incidence, risk factors, and chronobiology of acute myocardial infarction ten years after hurricane Katrina. Disaster Med Public Health Prep. 2018;12:1-6. This study documents the impact of a natural disaster (Hurricane Katrina) on the health and mental health of survivors.

11. Dai W, Chen L, Lai Z, Wang J, Liu A. The incidence of posttraumatic stress disorder among survivors after earthquakes: a systematic review and meta-analysis. BMC Psychiatry. 2016;16:188.

12. Murakami H, Hidechika A, Noda S, Mizoue T, Okazaki O, Ouchi $\mathrm{Y}$, et al. A cross-sectional survey of blood pressure of coastal city resident victims of the 2011 Tohoku tsunami. Am J Hypertens. 2013;26(6):799-807.

13. Tsujiuchi T, Yamaguchi M, Masuda K, Tsuchida M, Inomata T, Kumano H, et al. High prevalence of post-traumatic stress symptoms in relation to social factors in affected population one year after the Fukushima nuclear disaster. PLoS One. 2016;11(3): e0151807.

14.• Alfanso CA. PTSD and suicide after natural disasters. Psychiatric Times. 2018; This paper documents the increase in suicides following a natural disaster (Hurricane Maria in Puerto Rico).

15. Kessler RC, Galea S, Gruber MJ, Sampson NA, Ursano RJ, Wessely S. Trends in mental illness and suicidality after Hurricane Katrina. Mol Psychiatry. 2008 Apr;13(4):374-84.

16. Jefee-Bahloul H, Moustafa MK, Shebl FM, Barkil-Oteo A. Pilot assessment and survey of Syrian refugees' psychological stress and openness to referral for telepsychiatry (PASSPORT Study). Telemed J E Health. 2014;20(10):977-9. This study documents the rates of PTSD in Syrian refugees living in a UNHCR refugee camp in Turkey.

17. Cairncross F. The death of distance: Harvard Business School Press; 1997.

18. Tiberi O. Mental health in Haiti: beyond disaster relief. J Glob Health. 2016;1

19. Augusterfer EF, Mollica RF, Lavelle J. A review of telemental health in international and post-disaster settings. Int Rev Psychiatry. 2015;27(6):540-6.

20. Qadir TF, Fatima J, Usmani MH, Hussain SA. Telepsychiatry in Pakistan after natural disasters. Lancet Psychiatry. 2016;3(11): 1016.

21. Yellowlees P, Burke MM, Marks SL, Hilty DM, Shore JH. Emergency telepsychiatry. J Telemed Telecare. 2008;14(6):27781. 
22. Munro A, Kovats RS, Rubin GJ, Waite TD, Bone A, Armstrong B. Effect of evacuation and displacement on the association between flooding and mental health outcomes: a cross-sectional analysis of UK survey data. Lancet Planet Health. 2017;1(4):e134 41. This study documents the mental health impact of forced displacement.

23. United Nations High Commissioner on Refugees. 19 April 2018.

24. Nassan M, Frye MA, Adi A, Alarcon RD. Telepsychiatry for posttraumatic stress disorder: a call for action in the Syrian conflict. Lancet Psychiatry. 2015;2(10):866. This study documents the value of telepsychiatry in an active combat setting (Syrian conflict).

25. Jefee-Bahloul H, Barkil-Oteo A, Shukair N, Alraas W, Mahasneh $\mathrm{W}$. Using a store-and-forward system to provide global telemental heath supervision and training: a case from Syria. Acad Psychiatry. 2016;40(4):707-9.

26. Augusterfer EF. (Author's first-hand report from meeting with the Minister of Health Haiti). 2014.

27. Brandon AR, Song L, Deal AM, Gellin M, Sherwood E, Rosenstein DL. Using telehealth to train providers of a cancer support intervention. Telemedicine e-Health. 2015;21(10):793800 . This study documents the efficacy of telehealth as a medium to train providers in distant locations.
28. Mollica RF, Lopes Cardozo B, Osofsky HJ, Raphael B, Ager A, Salama P. Mental health in complex emergencies. Lancet. 2004;364(9450):2058-67. This study reports on the need for a self-care program for providers that include identification of risk factors and opportunities for resiliency.

29. Garbern SC, Ebbeling LG, Bartels SA. A systematic review of health outcomes among disaster and humanitarian responders. Prehosp Disaster Med. 2016;31(6):635-42. This study reports that mental health disorders among responders are higher and vary more than previously thought.

30. Naslund JA, Aschbrenner KA, Araya R, Marsch LA, Unutzer J, Patel V, et al. Digital technology for treating and preventing mental disorders in low-income and middle-income countries: a narrative review of the literature. Lancet Psychiatry. 2017;4(6):486-500. This review examines the use of digital technology for treating and preventing mental health disorders in globally underserved settings.

31. Latifi R, Tilley E. Telemedicine for disaster management: can it transform chaos into organized, structured care from a distance? Am J Disaster Med. 2014;9(1) 